\title{
Dispersion von Röntgenstrahlen im Gebiet der K-Kante und der L-Kanten schwerer Atome
}

\author{
H. Eisenlohr u. G. L. MÜller
}

Aus dem Institut für theoretische Physik der Universität Freiburg i. Br.

(Z. Naturforschg. 8a, 429—432 [1953]; eingegangen am ๖0. Mai 1953)

\begin{abstract}
Die von den Verfassern auf Grund der wellenmechanischen Dispersionstheorie berechnete Röntgenstreuung an schweren Atomen für einen geschlossenen Spektralbereich von sehr kurzwelliger Strahlung bis einschließlich der K- und L-Absorptionskanten wird an Hand zweier Beispiele für Tellur $(Z=52)$ und Wolfram $(Z=74)$ diskutiert und mit Meßwerten verglichen. Die als Ergebnis der Untersuchungen gewonnenen Streukurven zeigen systematische Abweichungen gegenüber früher errechneten und zum Teil gute Übereinstimmung mit der Erfahrung.
\end{abstract}

$\mathrm{D}$ ie ersten klassischen Ansätze zur Behandlung des Problems der kohärenten Röntgenstreuung durch amorphe Stoffe gingen von geometrischinterferenzmäßigen Überlegungen aus und führten zu Streuformeln, die gute Übereinstimmung mit der Erfahrung zeigten, solange man eine Röntgenstrahlung von genügend kurzer Wellenlänge benutzte. Im Bereich langwelliger Röntgenstrahlung und insbesondere in der Nähe von Absorptionskanten, wo die Bindungskräfte der Ladungen im Atom eine Rolle spielen, konnte die Streuwirkung auf Grund der einfachen interferenzmäßigen Vorstellung jedoch nicht mehr richtig dargestellt werden, so daß es sich als notwendig ergab, die wellenmechanische Dispersionstheorie heranzuziehen. Diese geht von der Wallerschen Streuformel aus. Nach ihr ist das für die kohärente Streustrahlung maßgebende Moment $\mathfrak{P}=\frac{e^{2}}{8 \pi^{2} m v^{2}}\left\{F_{0} \cdot \mathfrak{E}_{0}-\subseteq\right\} \cdot e^{2 \pi i v t}+$ konj. Glieder.

$\mathfrak{B}$ besteht somit aus 2 Summanden. Der erste, proportional $F_{0} \cdot \mathfrak{F}_{0}$ mit dem Atomamplitudenfaktor

$$
F_{0}=\sum_{s} \int \psi_{\varrho}^{*} e^{i\left(k-k_{0}, \mathrm{r}_{s}\right)} \psi_{\varrho} \mathrm{d} \tau,
$$

entspricht der geometrisch-interferenzmäßigen Theorie (Streuwirkung einer Ladungswolke) und wird allein maßgebend für hohe Frequenzen. Der zweite Bestandteil von $\mathfrak{B}$, proportional

$$
\begin{aligned}
& \subseteq=\frac{h}{4 \pi^{2} m} \sum_{\varrho^{\prime}}\left\{\frac{\left(\mathfrak{F}_{0} \mathfrak{A}^{0} \varrho_{\varrho^{\prime} \varrho}\right) \cdot \mathfrak{A}^{*} \varrho^{\prime} \varrho}{v_{\varrho^{\prime} \varrho}-v}\right.
\end{aligned}
$$

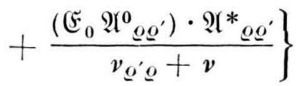

und den Matrixelementen:

$$
\begin{aligned}
& \mathfrak{A}_{\varrho \varrho^{\prime}}=\sum_{s} \int \psi_{\varrho^{\prime}}^{*} \cdot e^{-i\left(k_{0}, \mathrm{r}_{s}\right)} \cdot \operatorname{grad}_{s} \psi_{\varrho} \mathrm{d} \tau, \\
& \mathfrak{A}_{\varrho \varrho^{\prime}}^{*}=\sum_{s} \int \psi_{\varrho^{\prime}} \cdot e^{i\left(k, \mathrm{r}_{s}\right)} \cdot \operatorname{grad}_{s} \psi_{\varrho}^{*} \mathrm{~d} \tau,
\end{aligned}
$$

trägt der anomalen Dispersion Rechnung (siehe Resonanznenner) und wird in der Nähe der Absorptionskanten maßgebend. (Die $\nu^{\prime} \varrho^{\prime} \varrho$ sind die zum Übergang vom Grundzustand in den Anregungszustand gehörenden Frequenzen; $s$ ist Elektronenindex, $k_{0}, k$ sind die Ausbreitungsvektoren der einfallenden bzw. der gestreuten Röntgenwelle; die Eigenfunktionen $\psi_{\varrho}, \psi_{\varrho^{\prime}}$ sowie $\mathrm{d} \tau$ sind hochdimensional.)

$\mathrm{Hön} \mathbf{l}^{1}$ hat nun in einer früheren Arbeit gezeigt, wie man den ,,anomalen Dispersionsteil“ für eine Atomschale eines gegebenen Elementes berechnen kann und hat die Rechnungen speziell für die KElektronen explizite durchgeführt. Das Streumoment des Atoms (1) wird hierbei nach Multipolen entwickelt und die zu jedem Multipol gehörende charakteristische Frequenzabhängigkeit bestimmt. Bei der genauen Durchführung der Rechnung findet Hönl, daß zur Deutung der ,,anomalen Dispersion" der K-Schale die Berücksichtigung der Dipolglieder praktisch bereits vollständig ausreicht. Die berechneten Dispersionskurven, die von der Kallmann-Markschen wesentlich abweichen, zeigten gute Übereinstimmung mit den damals vorliegenden Meßergebnissen.

Von den Verfassern wurde nun kürzlich die Hönlsche Methode auf die L-Elektronenschale schwerer Atome übertragen. Es gelang auch hier, das Strah-

\footnotetext{
${ }^{1}$ H. Hönl, Ann. Physik. 18, 625 [1933].
} 
lungsfeld des streuenden Atoms nach elektrischen Multipolen zu entwickeln und jedem Multipol eine spezifische Frequenzabhängigkeit zuzuordnen. Als Ergebnis dieser sehr umfangreichen Rechnungen wurde für den Atomfaktor $F^{2}$ (Streuintensität des Atoms bezogen auf die eines freien ,,Thomson-Elektrons ") folgender summarischer Ausdruck gefunden:

$$
F^{2}=F_{0}{ }^{2}+\Delta_{1}+\Delta_{2} \text {. }
$$

Hierin bedeutet ${F_{0}}{ }^{2}$ den reduzierten idealen Atomfaktor, der im wesentlichen die ,,normale Dispersion" beschreibt und für den in der Nähe der LAbsorptionskanten $F_{0}{ }^{\prime}=F_{0}-n_{\mathrm{K}}$ gilt; $F_{0}$ ist der ,,ideale Atomfaktor" nach der geometrisch-interferenzmäßigen Theorie, $n_{\mathrm{K}}$ die ,ZZahl der Dispersionselektronen" der K-Schale. Die beiden Glieder $\Delta_{1}$ und $\Delta_{2}$ enthalten die ,,anomale Dispersion" $\Delta_{1}$ rührt allein von einem Dipol her, $\Delta_{2}$ ist ein gemischtes Dipol-Quadrupolglied.

Der zur numerischen Berechnung der Streukurven notwendige Rechenaufwand ist recht erheblich, so daß die Streuformeln bisher nur auf zwei Streusubstanzen, Tellur und Wolfram, angewandt werden konnten. Für Tellur wurden Winkel- und Frequenzabhängigkeit der Streustrahlung getrennt untersucht, beim Wolfram beschränkten wir uns auf die Diskussion der Frequenzabhängigkeit. Die Untersuchungsergebnisse sind im einzelnen folgende:

1. Die Winkelabhängigkeit der Streustrahlung des amorphen Tellur wurde für drei zwischen K- und LKante gelegene Wellenlängen berechnet. Abb.1 zeigt den Verlauf des Atomfaktors $F^{2}$ als Funktion von $\sin \theta / \lambda(\theta=$ Braggscher Reflektionswinkel $=$ halber Streuwinkel) beidiesendrei fest vorgegebenen Wellenlängen im Vergleich mit dem reduzierten idealen Atomfaktor $\left(F_{0}-1,2\right)^{2}$, wobei $n_{\mathrm{K}}$ als , Zahl der Dispersionselektronen" für Tellur einer Arbeit von Hönl² entnommen wurde. Wie zu erwarten, liefert die Theorie die größte durch ,,anomale Dispersion" verursachte Abweichung vom idealen Atomfaktor bei der $\mathrm{Cr}_{\mathrm{K}}$-Strahlung, deren Wellenlänge der L-Kante am nächsten liegt. Bei der $\mathrm{Cu}_{K^{-}}$und $\mathrm{Ag}_{K^{-}}$Strahlung ist die Abweichung vom idealen Atomfaktor geringer, außerdem erscheint sie hier mit entgegengesetztem Vorzeichen; wir befinden uns hier in dem Gebiet der durch die ,,anomale Dispersion" der L-Schale verursachten Überhöhung des Atomfaktors (siehe unten). Die Abweichung des nach unseren Rechnungen gewonnenen Atomfak-

${ }^{2}$ H. Hönl, Z. Physik 84, 15, Abb. 2 [1933]. tors vom idealen wird in der Hauptsache vom Dipolglied $\Delta_{1}$ getragen. Der Einfluß vom gemischten Dipol-Quadrupol-Glied $\Delta_{2}$ bleibt gegenüber $\Delta_{1}$ sehr klein, nimmt mit kürzer werdenden Wellenlängen zu und erreicht im extremsten Fall bei der AgStrahlung maximal $10 \%$ des $\Delta_{1}$-Wertes.

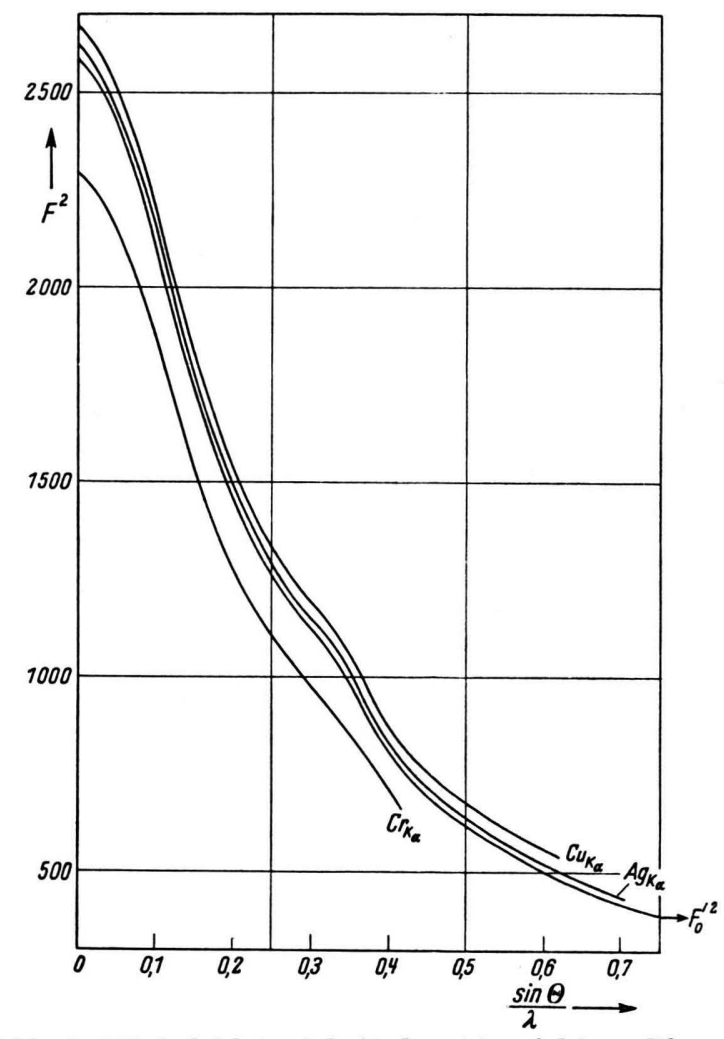

Abb. 1. Winkelabhängigkeit des Atomfaktors $F^{2}$ von Tellur $(Z=52)$ für $\operatorname{Cr}_{\alpha}$-Strahlung $(\lambda=2,28 \mathrm{AE})$, CuK ${ }_{\alpha}$-Strahlung $(\lambda=1,54 \mathrm{AE})$ und $\mathrm{Ag}_{\alpha_{\alpha}}$-Strahlung $(\lambda=0,588$ AE) im Vergleich mit dem reduzierten idealen Atomfaktor $F^{\prime 2}$.

2. Bei der Diskussion der Frequenzabhängigkeit des Atomfaktors beschränkten wir uns auf die Auswertung des Dipolgliedes $\Delta_{1}$, da $\Delta_{2}$ - wie bereits bemerkt - außerhalb eines sehr engen Spektralbereichs um die L-Kanten und für nicht allzu kurzwellige Strahlung keinen nennenswerten Beitrag liefert. Der Einfluß höherer Schalen konnte auf Grund vorliegender Messungen der Photoabsorptionskoeffizienten auf der langwelligen Seite der L-Kanten teilweise mitberücksichtigt werden. Bei Tellur stützten wir uns auf Absorptionsmessungen von Biermann ${ }^{3}$; für Wolfram mußten wir, da keine

${ }^{3}$ H. Biermann, Ann. Physik 26, 740 [1936]. 
geeigneten Messungen vorliegen, auf das von Jönsson ${ }^{4}$ gefundene Absorptionsgesetz zurückgreifen.

Die Dispersionskurven der Abb. 2 und 3, die den Atomamplitudenfaktor $\boldsymbol{F}$ in Abhängigkeit von der
Wellenlänge beim Streuwinkel $\theta=0$ (Richtung des Primärstrahles) wiedergeben, zeigen qualitativ etwa denselben Verlauf. Während aber die Hönlsche Dispersionskurve (gestrichelt) auf der langwelligen Seite der K-Kante asymptotisch in den

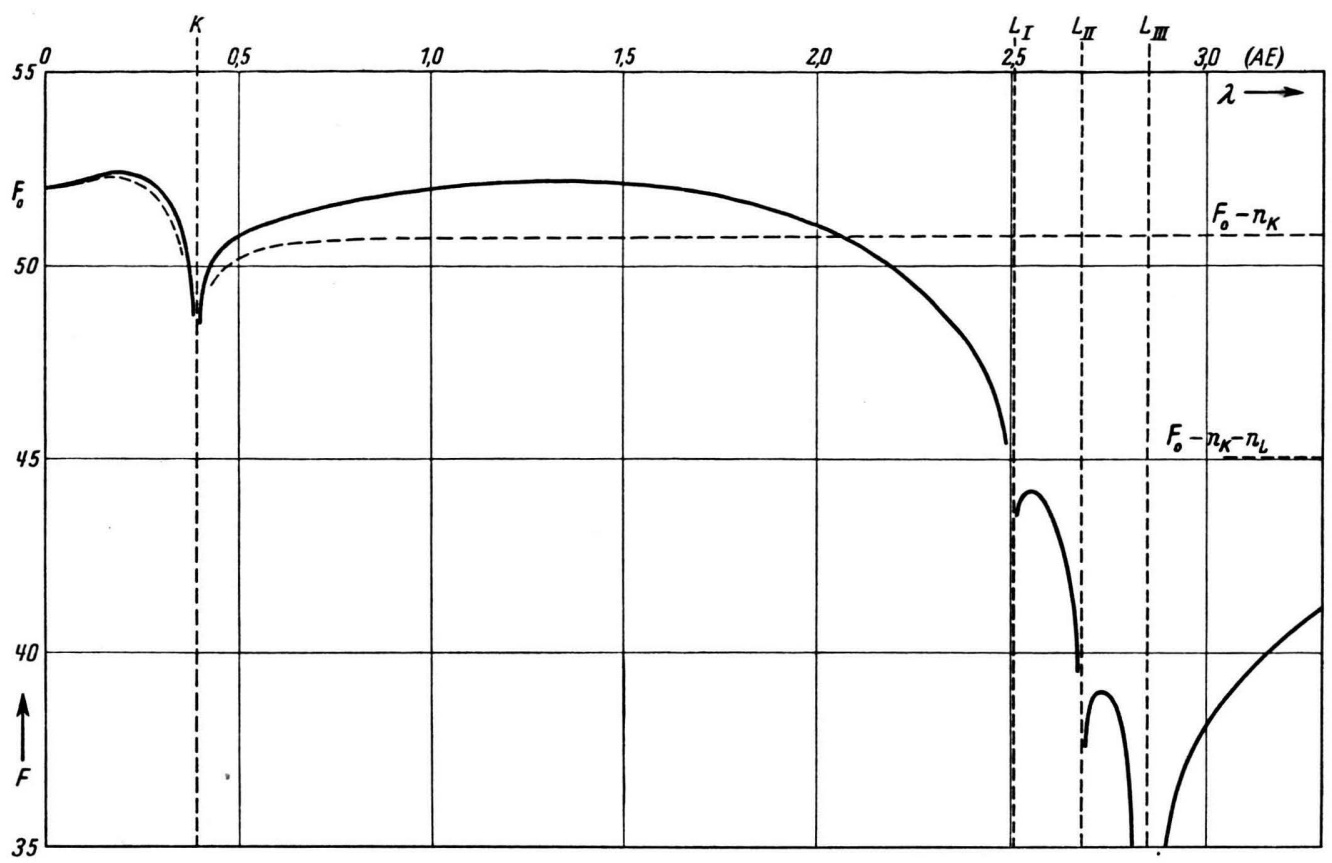

Abb. 2. Dispersionskurve für Tellur $(Z=52)$. Ausgezogene Kurve = Atomfaktor $F$ als Funktion der Wellenlänge, gestrichelte Kurve $=$ Hönlsche Dispersionskurve für den K-Einfluß.

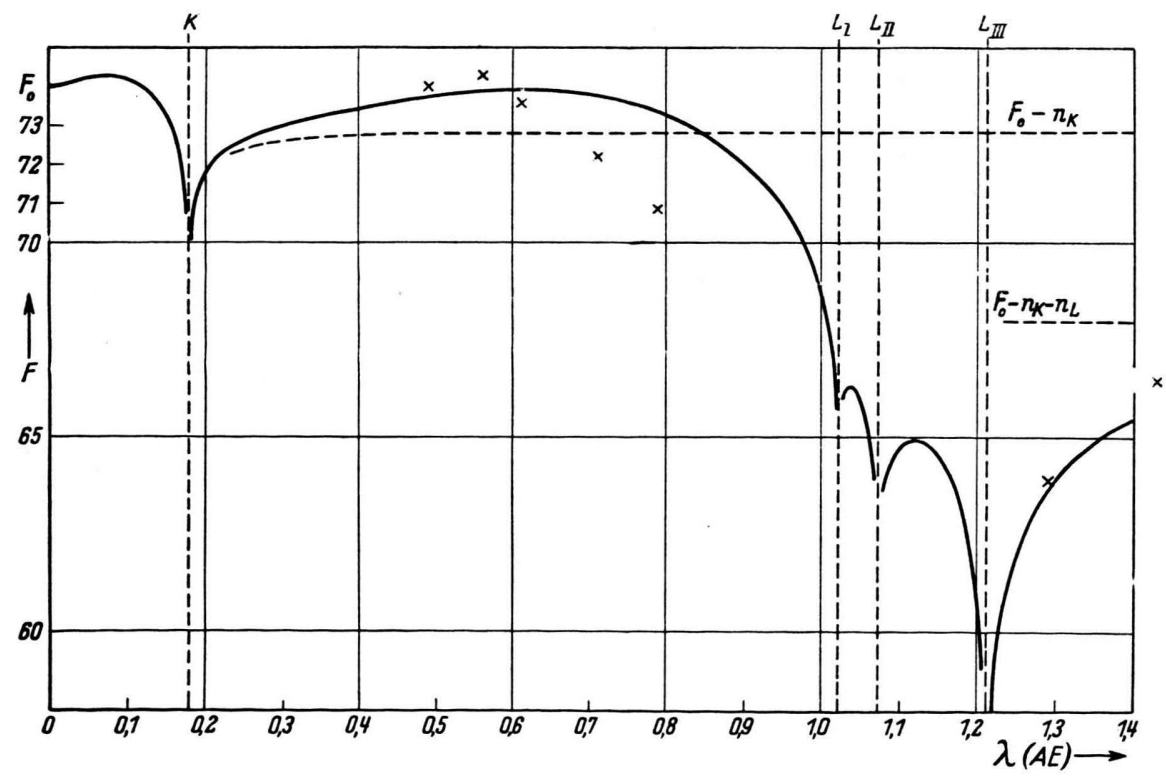

4 E. Jönsson, Dissertation Uppsala 1928/2.
Abb. 3. Dispersionskurve fürWolfram $(Z=74)$. Ausgezogene Kurve = Atomfaktor $F$ in Abhängigkeit von der Wellenlänge, gestrichelte Kurve = langwelliger Ast der Hönlschen Dispersionskurve. $\times \times \times$ Meßwerte von Brentano und Baxter. 
konstanten Wert $F_{0}-n_{\mathrm{K}}$ ausläuft, steigen unsere Dispersionskurven weit über diesen Wert hinaus und erreichen zwischen der K-Kante und den L-Kanten ein Maximum, das beim Tellur höher als $F_{0}$ liegt und beim Wolfram beinahe bis an diese Größe heranreicht. Es scheint daher nicht möglich, die Zahl der K-Dispersionselektronen in der bisherigen einfachen Weise experimentell zu bestimmen, wie dies ohne Kenntnis des Einflusses der anomalen L-Dispersion versucht worden ist ${ }^{5}$. Während für Tellur noch keine experimentellen Werte der Dispersion vorliegen, haben Brentano und Baxter ${ }^{6}$ bereits vor längerer Zeit Dispersionsmessungen an Wolfram in der Nähe der L-Kanten ausgeführt. Diese (durch $x \times$ in das Wolfram-Diagramm eingetragen) bestätigen die von uns gefundene Überhöhung des Dispersionsverlaufs zwischen der K- und den L-Kanten. Die beiden Meßpunkte in der Nähe der $\mathrm{L}_{\mathrm{I}}$-Kante liegen allerdings etwas zu tief, während die beiden Meßwerte auf der langwelligen Seite der L-Kanten

${ }^{5}$ Vgl. R. W. James, The Optical Principles of the Diffraction of X-Rays, London 1950. wieder befriedigend mit unserer Kurve übereinstimmen. Man beachte, daß in diesen langwelligen Spektralbereich bereits die anomale Dispersion der M-Schale einwirkt, die in der vorliegenden Untersuchung nur teilweise berücksichtigt werden konnte. Die bezüglich der gesamten anomalen M-Dispersion korrigierte Dispersionskurve würde analog den oben besprochenen Verhältnissen an der K-Kante nach höheren $F$-Werten hin verschoben werden, also zugunsten der beiden auf der langwelligen Seite der L-Kanten gefundenen experimentellen Werte.

Eine ausführliche Mitteilung mit Darlegung des Rechenganges erfolgt demnächst in der ,Zeitschrift für Physik".

Herrn Prof. H. Hönl danken wir an dieser Stelle für die Anregung zu dieser Arbeit und sein förderndes Interesse. Für die großzügige Unterstützung der Arbeit durch die Deutsche Forschungsgemeinschaft sei dieser ebenfalls bestens gedankt.

6 J. Brentano u. A. Baxter, Z. Physik 89, 720 [1934].

\title{
Elektronenzustände kubischer Kristalle in Brillouinscher Näherung*
}

\author{
Von J. Homilius \\ Aus dem Institut für theoretische Physik der Universität Münster i. Westf.
}

(Z. Naturforschg. 8a, 432-441 [1953]; eingegangen am 29. Mai 1953)

\begin{abstract}
Eigenfunktionen und Energieflächen für die kubischen Gitter $\Gamma_{c}, \Gamma_{c}^{\prime}$ und $\Gamma_{c}{ }^{\prime \prime}$ werden in erster Brillouinscher Näherung behandelt und insbesondere für das kubische Flächengitter näher untersucht. Es werden die Energieaufspaltungen in den Umgebungen der ersten sechs Resonanzstellen berechnet und Energiebänder längs charakteristischer Geraden auf den Begrenzungsflächen der ersten Brillouin-Zonen graphisch dargestellt.
\end{abstract}

$Z^{n}$ ur Berechnung der inneren Feldemission in Einkristallen nach dem Houstonschen Ansatz ${ }^{1,2}$ ist die Kenntnis der Elektroneneigenfunktionen im Kristall und besonders der dazugehörigen Energiewerte in Abhängigkeit von den Impulsen bzw. Wellenzahlen nötig, und zwar kommt es besonders auf die Verhältnisse an den Bandgrenzen an. Aus den vorhandenen Rechnungen lassen sich meist nur der Energieverlauf längs Geraden, welche innerhalb der Zonen liegen, und die Wellenfunktion fast stets nur

* Erster Teilauszug aus der Dissertation, Münster i. Westf.

1 W. V. Houston, Physic. Rev. 57, 184 [1940]. für bestimmte Punkte des Wellenzahlraumes entnehmen. Eine für den ganzen Wellenzahlraum gültige Lösung wurde von Slater ${ }^{3}$ angegeben, doch ist sein Potentialansatz zu speziell, da er nur Fourier-Koeffizienten $v_{100}$ enthält; die resultierenden Funktionen sind für unsere Zwecke zu schwer zu handhaben. Um Wellenfunktionen und Energieflächen in einer möglichst einfachen analytischen Gestalt zu gewinnen, welche die etwas komplizierten Operationen der Houstonschen Theorie zuläßt,

\footnotetext{
2 J. Homilius, Z. Naturforschg. 7 a, 290 [1952].

${ }^{3}$ J. C. Slater, Physic. Rev. 87, 807 [1952].
} 